PROCEEDINGS OF THE

AMERICAN MATHEMATICAL SOCIETY

Volume 131, Number 5, Pages 1373-1383

S 0002-9939(02)06899-5

Article electronically published on December 6, 2002

\title{
INTERPOLATION FOR MULTIPLIERS ON REPRODUCING KERNEL HILBERT SPACES
}

\author{
VLADIMIR BOLOTNIKOV
}

(Communicated by Joseph A. Ball)

\begin{abstract}
All solutions of a tangential interpolation problem for contractive multipliers between two reproducing kernel Hilbert spaces of analytic vectorvalued functions are characterized in terms of certain positive kernels. In a special important case when the spaces consist of analytic functions on the unit ball of $\mathbb{C}^{d}$ and the reproducing kernels are of the form $\left(1-\langle z, w\rangle^{-1}\right) I_{p}$ and $(1-\langle z, w\rangle)^{-1} I_{q}$, the characterization leads to a parametrization of the set of all solutions in terms of a linear fractional transformation.
\end{abstract}

\section{INTRODUCTION}

A Hilbert space $\mathcal{H}$ of $\mathbb{C}^{p \times 1}$-valued functions which are defined on a domain $\Omega \in \mathbb{C}^{d}$ is said to be a reproducing kernel Hilbert space if there exists a $\mathbb{C}^{p \times p_{-}}$ valued function $K(z, \omega)$ such that for every point $\omega \in \Omega$ and every vector $c \in \mathbb{C}^{p}$, the function $K_{w} c:=K(\cdot, w) c$ belongs to $\mathcal{H}$ and $\left\langle f, K_{w} c\right\rangle_{\mathcal{H}}=c^{*} f(w)$ for every function $f \in \mathcal{H}$. The function $K(z, w)$ turns to be positive on $\Omega$ in the sense that $\sum_{j, \ell=1}^{n} c_{j}^{*} K\left(z^{(j)}, z^{(\ell)}\right) c_{\ell} \geq 0$ for every choice of an integer $n$, of vectors $c_{1}, \ldots, c_{n} \in$ $\mathbb{C}^{p}$ and of points $z^{(1)}, \ldots, z^{(n)} \in \Omega$ or, equivalently, if the Hermitian block matrix with $\ell j$-th entry $K\left(z^{(j)}, z^{(\ell)}\right)$ is positive semidefinite. This property will be denoted by $K(z, \omega) \succeq 0$. The function $K(z, \omega)$ is, furthermore, uniquely defined (as is easily verified), and is called the reproducing kernel of $\mathcal{H}$. The fundamental result of Aronszajn [4] states that for every positive kernel $K$ on $\Omega$, there is a unique reproducing kernel Hilbert space $\mathcal{H}(K)$ with $K$ as its reproducing kernel. Moreover, the set $\mathcal{H}_{0}$ consisting of functions of the form $\sum K\left(\cdot, w_{j}\right) c_{j}$, where $\left\{c_{j}\right\}$ and $\left\{w_{j}\right\}$ are finite sequences in $\mathbb{C}^{p}$ and $\Omega$, respectively, is a dense linear manifold in $\mathcal{H}(K)$. In what follows we shall write $K_{w}(z)$ rather than $K(z, w)$ if the last function will be considered as a function of $z$ with a fixed point $w \in \Omega$.

Let $K^{(1)}(z, w)$ and $K^{(2)}(z, w)$ be two positive kernels on $\Omega$, which are respectively, $\mathbb{C}^{q \times q_{-}}$and $\mathbb{C}^{p \times p}$-valued and let $\mathcal{H}\left(K^{(1)}\right)$ and $\mathcal{H}\left(K^{(2)}\right)$ be the corresponding reproducing kernel Hilbert spaces. A $\mathbb{C}^{p \times q}$-valued function $S$ defined on $\Omega$ is called a contractive multiplier from $\mathcal{H}\left(K^{(1)}\right)$ to $\mathcal{H}\left(K^{(2)}\right)$ if the multiplication operator $\mathbf{M}_{S}: \mathcal{H}\left(K^{(1)}\right) \rightarrow \mathcal{H}\left(K^{(2)}\right)$, defined by

$$
\mathbf{M}_{S}(f(z))=S(z) f(z),
$$

Received by the editors February 24, 2001 and, in revised form, March 23, 2001.

2000 Mathematics Subject Classification. Primary 41A05, 46E22.

(C)2002 American Mathematical Society 
is a contraction (if $K^{(1)}=K^{(2)}$, then $S$ is called a contractive multiplier on $\left.\mathcal{H}\left(K^{(1)}\right)\right)$. The latter means that $I_{\mathcal{H}\left(K^{(2)}\right)}-\mathbf{M}_{S} \mathbf{M}_{S}^{*} \geq 0$ and is equivalent to

$$
K_{S}(z, w):=K^{(2)}(z, w)-S(z) K^{(1)}(z, w) S(w)^{*} \succeq 0 \quad(z, w \in \Omega) .
$$

The set of all contractive multipliers $S$ from $\mathcal{H}\left(K^{(1)}\right)$ to $\mathcal{H}\left(K^{(2)}\right)$ will be denoted by $\mathcal{S}\left(K^{(1)}, K^{(2)}\right)$.

In this paper we shall focus on the following interpolation

Problem 1.1. Given functions $f_{1}, \ldots, f_{n}$ in $\mathcal{H}\left(K^{(1)}\right)$ and $h_{1}, \ldots, h_{n}$ in $\mathcal{H}\left(K^{(2)}\right)$ find necessary and sufficient conditions which insure the existence of a function $S \in \mathcal{S}\left(K^{(1)}, K^{(2)}\right)$ such that

$$
\left(\mathbf{M}_{S}^{*} h_{j}\right)(z)=f_{j}(z), \quad j=1, \ldots, n .
$$

We shall make frequent use of notations

$$
H(z)=\left[\begin{array}{llll}
h_{1}(z) & \ldots & h_{n}(z)
\end{array}\right] \text { and } F(z)=\left[\begin{array}{lll}
f_{1}(z) & \ldots & f_{n}(z)
\end{array}\right]
$$

which allows us to rewrite interpolation conditions (1.3) in a more compact form as

$$
\left(\mathbf{M}_{S}^{*} H\right)(z)=F(z) .
$$

Note that the tangential Nevanlinna-Pick problem in the class $\mathcal{S}\left(K^{(1)}, K^{(2)}\right)$ is a particular case of Problem 1.1. Indeed, a simple computation shows that

$$
\left(\mathbf{M}_{S}^{*} K_{w}^{(2)}\right)(z)=K_{w}^{(1)}(z) S(w)^{*}
$$

and thus a special choice of $h_{j}=K_{w_{j}}^{(2)} c_{j}$ and $f_{j}=K_{w_{j}}^{(1)} d_{j}$ in (1.3) leads to the left-sided interpolation conditions

$$
K_{w_{j}}^{(1)}(z) S\left(w_{j}\right)^{*} c_{j} \equiv K_{w_{j}}^{(1)}(z) d_{j}, \quad j=1, \ldots, n .
$$

Under the further assumption that $K^{(1)}$ is not degenerate (i.e., $K^{(1)}(z, z)>0$ for all $z \in \Omega$ ), the latter conditions are equivalent to the classical Nevanlinna-Pick conditions

$$
S\left(w_{j}\right)^{*} c_{j}=d_{j}, \quad j=1, \ldots, n .
$$

In Section 2 all the solutions $S$ of Problem 1.1 are characterized in terms of certain positive kernels constructed from the interpolation data. In Section 3 we consider a particular case of Problem 1.1 for multipliers on multivariable analogues of the Hardy space $\mathbf{H}_{2}$ of the unit disk, studied in [5]. For this case, the general result (Theorem 2.4 leads to a parametrization of the set of all solutions in terms of a linear fractional transformation. The Nevanlinna-Pick problem in this setting (see Remark 3.1] has been considered in [6].

\section{THE MAIN RESULT}

As mentioned above, for a function $S \in \mathcal{S}\left(K^{(1)}, K^{(2)}\right)$ the kernel $K_{S}(z, w)$ defined in (1.2) is positive on $\Omega$. The corresponding reproducing kernel Hilbert space will be referred to as $\mathcal{H}(S)$.

The original characterization of $\mathcal{H}(S)$, as the space of all elements functions $f \in \mathcal{H}\left(K^{(2)}\right)$ such that

$$
\kappa(f):=\sup _{g \in \mathcal{H}\left(K^{(1)}\right)}\left\{\|f+S g\|_{\mathcal{H}\left(K^{(2)}\right)}^{2}-\|g\|_{\mathcal{H}\left(K^{(1)}\right)}^{2}\right\}
$$

is finite and $\|f\|_{\mathcal{H}(S)}^{2}=\kappa(f)$, is due to de Branges and Rovnyak [8]. 
On the other hand, the general complementation theory (see, e.g., [15, Ch.1]) applied to the contractive operator $\mathbf{M}_{S}$ provides the characterization of $\mathcal{H}(S)$ as the operator range

$$
\mathcal{H}(S)=\operatorname{Range}\left(I-\mathbf{M}_{S} \mathbf{M}_{S}^{*}\right)^{\frac{1}{2}} \text { with }\left\|\left(I-\mathbf{M}_{S} \mathbf{M}_{S}^{*}\right)^{\frac{1}{2}} h\right\|_{\mathcal{H}(S)}=\|(I-\pi) h\|_{\mathcal{H}_{\mathcal{H}\left(K^{(2)}\right)}},
$$

where $\pi$ denotes the orthogonal projection onto $\operatorname{Ker}\left(I-\mathbf{M}_{S} \mathbf{M}_{S}^{*}\right)^{\frac{1}{2}}$.

To state the main theorem we need some preliminary results. The first one follows immediately from the characterization (2.2) upon setting $h=\left(I-\mathbf{M}_{S} \mathbf{M}_{S}^{*}\right)^{\frac{1}{2}} f$.

Lemma 2.1. Let $S \in \mathcal{S}\left(K^{(1)}, K^{(2)}\right)$ and $f \in \mathcal{H}\left(K^{(2)}\right)$. Then $\left(I_{\mathcal{H}\left(K^{(2)}\right)}-\mathbf{M}_{S} \mathbf{M}_{S}^{*}\right) f$ belongs to $\mathcal{H}(S)$ and

$$
\left\|\left(I-\mathbf{M}_{S} \mathbf{M}_{S}^{*}\right) f\right\|_{\mathcal{H}(S)}^{2}=\left\langle\left(I-\mathbf{M}_{S} \mathbf{M}_{S}^{*}\right) f, f\right\rangle_{\mathcal{H}\left(K^{(2)}\right)} .
$$

Let $K$ be a positive $\mathbb{C}^{N \times N}$-valued kernel on $\Omega$ and let $\mathcal{H}(K)$ be the corresponding reproducing kernel Hilbert space consisting of $\mathbb{C}^{N}$-valued vector functions. The usage of matrix-valued functions with the columns in $\mathcal{H}(K)$ prompts us to introduce (besides the standard inner product) the following bilinear form:

$$
[X, Y]_{\mathcal{H}(K)}=\left(\left\langle x_{\ell}, y_{j}\right\rangle_{\mathcal{H}(K)}\right)_{\ell=1, \ldots, n}^{j=1, \ldots, m}
$$

which makes sense for every pair of functions

$$
X(z)=\left[x_{1}(z) \ldots x_{n}(z)\right] \in(\mathcal{H}(K))^{1 \times n}, Y(z)=\left[y_{1}(z) \ldots y_{m}(z)\right] \in(\mathcal{H}(K))^{1 \times m},
$$

which are respectively, $\mathbb{C}^{N \times n}$ - and $\mathbb{C}^{N \times m}$-valued.

Remark 2.2. The form (2.4) can be viewed as the matrix representation of the operator $\mathbf{M}_{Y}^{*} \mathbf{M}_{X}: \mathbb{C}^{n} \rightarrow \mathbb{C}^{m}$ with respect to the standard basis, where $\mathbf{M}_{X}$ : $\mathbb{C}^{n} \rightarrow \mathcal{H}(K)$ and $\mathbf{M}_{Y}: \mathbb{C}^{m} \rightarrow \mathcal{H}(K)$ are the multiplication operators given by

$$
\mathbf{M}_{X} c=X(z) c \quad \text { and } \quad \mathbf{M}_{Y} d=Y(z) d .
$$

The next preliminary lemma characterizes $\mathcal{H}(K)$ in terms of positive kernels (see [9, Theorem 2.2] for scalar-valued kernels and [2, Lemma 2.2] for the matrix case):

Lemma 2.3. A nonzero vector-valued function $f$ defined on $\Omega$ belongs to $\mathcal{H}(K)$ and satisfies $\|f\|_{\mathcal{H}(K)}^{2} \leq \gamma$ if and only if the kernel $K(z, w)-\gamma^{-1} f(z) f(w)^{*}$ is positive on $\Omega$.

The next theorem characterizes all the solutions $S$ of Problem [1.1 in terms of positive kernels and in terms of the reproducing kernel Hilbert spaces $\mathcal{H}(S)$. The first develops Potapov's method (which characterizes the solutions of an interpolation problem in terms of a related fundamental matrix inequality [11]), and the second is related to reproducing kernel methods in interpolation theory [10].

Theorem 2.4. Let $H \in\left(\mathcal{H}\left(K^{(2)}\right)\right)^{1 \times n}$ and $F \in\left(\mathcal{H}\left(K^{(1)}\right)\right)^{1 \times n}$ be as in [1.4), let $S$ be a $p \times q$ matrix-valued function which is analytic in $\Omega$, let $K_{S}$ be defined by (1.2) and let

$$
P:=[H, H]_{\mathcal{H}\left(K^{(2)}\right)}-[F, F]_{\mathcal{H}\left(K^{(1)}\right)}
$$

and

$$
B(z)=H(z)-S(z) F(z)
$$


Then the following statements are equivalent:

(1) $S$ is a solution to Problem 1.1

(2) For every choice of $x \in \mathbb{C}^{n}$, the function $B(z) x$ belongs to the space $\mathcal{H}(S)$ and

$$
\|B x\|_{\mathcal{H}(S)}^{2}=x^{*} P x .
$$

(3) The following kernel is positive on $\Omega$ :

$$
\mathbf{K}(z, w):=\left[\begin{array}{cc}
P & B(w)^{*} \\
B(z) & K_{S}(z, w)
\end{array}\right] \succeq 0 .
$$

(4) The following operator

$$
\mathbf{P}:=\left[\begin{array}{cc}
P & \mathbf{M}_{B}^{*} \\
\mathbf{M}_{B} & I-\mathbf{M}_{S} \mathbf{M}_{S}^{*}
\end{array}\right]:\left[\begin{array}{l}
\mathbb{C}^{n} \\
\mathcal{H}\left(K^{(2)}\right)
\end{array}\right] \rightarrow\left[\begin{array}{l}
\mathbb{C}^{n} \\
\mathcal{H}\left(K^{(1)}\right)
\end{array}\right]
$$

is positive semidefinite.

Proof. $(\mathbf{1}) \Rightarrow(\mathbf{2})$. Let $S$ be a solution to Problem 1.1. Then $\mathbf{M}_{S} \mathbf{M}_{S}^{*} \leq I_{\mathcal{H}\left(K^{(2)}\right)}$ and (1.5) is in force. Substituting (1.5) into the right-hand side of (2.5) and (2.6) we get

$$
P=[H, H]_{\mathcal{H}\left(K^{(2)}\right)}-\left[\mathbf{M}_{S}^{*} H, \mathbf{M}_{S}^{*} H\right]_{\mathcal{H}\left(K^{(1)}\right)}=\left[\left(I-\mathbf{M}_{S} \mathbf{M}_{S}^{*}\right) H, H\right]_{\mathcal{H}\left(K^{(2)}\right)}
$$

and

$$
B(z)=H(z)-S(z)\left(\mathbf{M}_{S}^{*} H\right)(z)=\left(\left\{I-\mathbf{M}_{S} \mathbf{M}_{S}^{*}\right\} H\right)(z) .
$$

Since $H x \in \mathcal{H}\left(K^{(2)}\right)$ for every $x \in \mathbb{C}^{n}$, the last formula implies, by Lemma 2.1 that $B x \in \mathcal{H}(S)$. Finally, by (2.3) and 2.10),

$$
\|B x\|_{\mathcal{H}(S)}^{2}=\left\|\left(I-\mathbf{M}_{S} \mathbf{M}_{S}^{*}\right) H x\right\|_{\mathcal{H}(S)}^{2}=\left\langle\left(I-\mathbf{M}_{S} \mathbf{M}_{S}^{*}\right) H x, H x\right\rangle_{\mathcal{H}\left(K^{(2)}\right)}=x^{*} P x .
$$

$(\mathbf{2}) \Rightarrow(\mathbf{3})$. By Lemma 2.3, equality (2.7) implies

$$
K_{S}(z, w)-\left(x^{*} P x\right)^{-1} B(z) x x^{*} B(w)^{*} \succeq 0 \quad(z, w \in \Omega)
$$

for every vector $x \in \mathbb{C}^{n}$ such that $P x \neq 0$. The last inequality is obviously equivalent to

$$
\left[\begin{array}{ll}
x^{*} P x & x^{*} B(w)^{*} \\
B(z) x & K_{S}(z, w)
\end{array}\right] \succeq 0 \quad(z, w \in \Omega) .
$$

If $P x=0$, then (2.7) implies $B(z) x \equiv 0$, and thus (2.12) is in force as well. Thus, (2.12) holds for every $x \in \mathbb{C}^{n}$, which is equivalent to (2.8).

$(3) \Rightarrow(4)$. By the reproducing kernel property,

$$
\left(\mathbf{M}_{S}^{*} K_{w}^{(2)}\right)(z)=\left[\mathbf{M}_{S}^{*} K_{w}^{(2)}, K_{z}^{(1)}\right]_{\mathcal{H}\left(K^{(1)}\right)}=\left[K_{w}^{(2)}, S K_{z}^{(1)}\right]_{\mathcal{H}\left(K^{(2)}\right)}=K_{w}^{(1)}(z) S(w)^{*}
$$

and therefore,

$$
\left[\left(I-\mathbf{M}_{S} \mathbf{M}_{S}^{*}\right) K_{w}^{(2)}, K_{z}^{(2)}\right]_{\mathcal{H}\left(K^{(2)}\right)}=K_{w}^{(2)}(z)-S(z) K_{w}^{(1)}(z) S(w)^{*}=K_{S}(z, w),
$$


which shows, in particular, that the kernel $K_{S}$ is positive. Fix a vector $f \in \mathbb{C}^{n} \oplus$ $\mathcal{H}\left(K^{(2)}\right)$ of the form

$$
f=\sum_{j=1}^{r}\left[\begin{array}{c}
c_{j} \\
K_{w^{(j)}}^{(2)} d_{j}
\end{array}\right] \quad\left(c_{j} \in \mathbb{C}^{n}, d_{j} \in \mathbb{C}^{p}, w^{(j)} \in \Omega\right) .
$$

By (2.13),

$$
\left\langle\left(I-\mathbf{M}_{S} \mathbf{M}_{S}^{*}\right) K_{w^{(\ell)}}^{(2)} d_{\ell}, K_{w^{(j)}}^{(2)} d_{j}\right\rangle_{\mathcal{H}\left(K^{(2)}\right)}=d_{j}^{*} K_{S}\left(w^{(j)}, w^{(\ell)}\right) d_{\ell}
$$

and by the reproducing kernel property,

$$
\left\langle\mathbf{M}_{B} c_{\ell}, K_{w^{(j)}}^{(2)} d_{j}\right\rangle_{\mathcal{H}\left(K^{(2)}\right)}=d_{j}^{*} B\left(w^{(j)}\right) c_{\ell} .
$$

Using the two last equalities and taking into account partitionings (2.8) and (2.9) of $\mathbf{K}$ and $\mathbf{P}$ we get

$$
\left\langle\mathbf{P}\left[\begin{array}{c}
c_{\ell}^{(2)} \\
K_{w^{(\ell)}}^{(2)} d_{j}
\end{array}\right],\left[\begin{array}{c}
c_{j} \\
K_{w^{(j)}}^{(2)} d_{j}
\end{array}\right]\right\rangle_{\mathbb{C}^{n} \oplus \mathcal{H}\left(K^{(2)}\right)}=\left[\begin{array}{ll}
c_{j}^{*} & d_{j}^{*}
\end{array}\right] \mathbf{K}\left(w^{(j)}, w^{(\ell)}\right)\left[\begin{array}{c}
c_{\ell} \\
d_{\ell}
\end{array}\right] .
$$

By linearity and in view of (2.14),

$$
\langle\mathbf{P} f, f\rangle_{\mathbb{C}^{n} \oplus \mathcal{H}\left(K^{(2)}\right)}=\sum_{j, \ell=1}^{r}\left[\begin{array}{cc}
c_{j}^{*} & d_{j}^{*}
\end{array}\right] \mathbf{K}\left(w^{(j)}, w^{(\ell)}\right)\left[\begin{array}{c}
c_{\ell} \\
d_{\ell}
\end{array}\right] .
$$

Since the kernel $\mathbf{K}(z, w)$ is positive on $\Omega$, the expression on the right-hand side of the last equality is nonnegative. Thus, $\langle\mathbf{P} f, f\rangle_{\mathbb{C}^{n} \oplus \mathcal{H}\left(K^{(2)}\right)} \geq 0$ for every vector $f$ of the form (2.14). Since the set of all such vectors is dense in $\mathbb{C}^{n} \oplus \mathcal{H}\left(K^{(2)}\right), \mathbf{P}$ is positive semidefinite.

$(\mathbf{4}) \Rightarrow(\mathbf{1})$. If $\mathbf{P}$ is positive semidefinite, then in particular, $\mathbf{M}_{S} \mathbf{M}_{S}^{*} \leq I$ and therefore, $S \in \mathcal{S}\left(K^{(1)}, K^{(2)}\right)$. It remains to show that the interpolation condition (1.5) is valid. To this end let us consider the block operator

$$
\widehat{\mathbf{P}}=\left[\begin{array}{lll}
I_{\mathcal{H}\left(K^{(1)}\right)} & \mathbf{M}_{F} & \mathbf{M}_{S}^{*} \\
\mathbf{M}_{F}^{*} & \mathbf{M}_{H}^{*} \mathbf{M}_{H} & \mathbf{M}_{H}^{*} \\
\mathbf{M}_{S} & \mathbf{M}_{H} & I_{\mathcal{H}\left(K^{(2)}\right)}
\end{array}\right]:\left[\begin{array}{l}
\mathcal{H}\left(K^{(1)}\right) \\
\mathbb{C}^{n} \\
\mathcal{H}\left(K^{(2)}\right)
\end{array}\right] \rightarrow\left[\begin{array}{l}
\mathcal{H}\left(K^{(1)}\right) \\
\mathbb{C}^{n} \\
\mathcal{H}\left(K^{(2)}\right)
\end{array}\right]
$$

Here we use a somewhat sloppy notation: the domain and range of a multiplication operator $\mathbf{M}_{X}$ depends on the $X$. Specifically, we have

$$
\mathbf{M}_{F}: \mathbb{C}^{n} \rightarrow \mathcal{H}\left(K^{(1)}\right), \quad \mathbf{M}_{H}: \mathbb{C}^{n} \rightarrow \mathcal{H}\left(K^{(2)}\right)
$$

with therefore

$$
\mathbf{M}_{F}^{*}: \mathcal{H}\left(K^{(1)}\right) \rightarrow \mathbb{C}^{n}, \quad \mathbf{M}_{H}^{*}: \mathcal{H}\left(K^{(2)}\right) \rightarrow \mathbb{C}^{n}
$$

but

with therefore

$$
\mathbf{M}_{S}: \mathcal{H}\left(K^{(1)}\right) \rightarrow \mathcal{H}\left(K^{(2)}\right)
$$

$$
\mathbf{M}_{S}^{*}: \mathcal{H}\left(K^{(2)}\right) \rightarrow \mathcal{H}\left(K^{(1)}\right) .
$$

Taking advantage of Remark 2.2 we obtain from (2.5) and (2.6) the representations

$$
P=\mathbf{M}_{H}^{*} \mathbf{M}_{H}-\mathbf{M}_{F}^{*} \mathbf{M}_{F}, \quad \mathbf{M}_{B}=\mathbf{M}_{H}-\mathbf{M}_{S} \mathbf{M}_{F},
$$


which allow us to conclude that the operator $\mathbf{P}$ given in (2.9) is the Schur complement of the $(1,1)$ block entry of $\widehat{\mathbf{P}}$ :

$$
\mathbf{P}=\left[\begin{array}{cl}
\mathbf{M}_{H}^{*} \mathbf{M}_{H} & \mathbf{M}_{H}^{*} \\
\mathbf{M}_{H} & I_{\mathcal{H}\left(K^{(2)}\right)}
\end{array}\right]-\left[\begin{array}{l}
\mathbf{M}_{F}^{*} \\
\mathbf{M}_{S}
\end{array}\right]\left[\begin{array}{ll}
\mathbf{M}_{F} & \mathbf{M}_{S}^{*}
\end{array}\right]
$$

Since $\mathbf{P}$ is positive semidefinite, it follows that $\widehat{\mathbf{P}} \geq 0$. Therefore the Schur complement of the $(3,3)$ block entry of $\widehat{\mathbf{P}}$ is positive semidefinite:

$$
\begin{aligned}
& {\left[\begin{array}{cc}
I_{\mathcal{H}\left(K^{(1)}\right)} & \mathbf{M}_{F} \\
\mathbf{M}_{F}^{*} & \mathbf{M}_{H}^{*} \mathbf{M}_{H}
\end{array}\right]-\left[\begin{array}{c}
\mathbf{M}_{S}^{*} \\
\mathbf{M}_{H}^{*}
\end{array}\right]\left[\begin{array}{ll}
\mathbf{M}_{S} & \mathbf{M}_{H}
\end{array}\right]} \\
& \quad=\left[\begin{array}{cc}
I-\mathbf{M}_{S}^{*} \mathbf{M}_{S} & \mathbf{M}_{F}-\mathbf{M}_{S}^{*} \mathbf{M}_{H} \\
\mathbf{M}_{F}^{*}-\mathbf{M}_{H}^{*} \mathbf{M}_{S} & 0
\end{array}\right] \geq 0 .
\end{aligned}
$$

The last relation implies that $\mathbf{M}_{F}-\mathbf{M}_{S}^{*} \mathbf{M}_{H}=0$, which is equivalent to (1.5).

\section{EXAMPLE}

In this section we apply the preceding analysis to a class $\mathcal{S}^{p \times q}$ of $\mathbb{C}^{p \times q}$-valued functions $S$ analytic in the unit ball $\mathbb{B}^{d}=\left\{z=\left(z_{1}, \ldots, z_{d}\right) \in \mathbb{C}^{d}: \sum_{1}^{d}\left|z_{j}\right|^{2}<1\right\}$ of $\mathbb{C}^{d}$ and such that

$$
K_{S}(z, w)=\frac{I_{p}-S(z) S(w)^{*}}{1-\langle z, w\rangle} \succeq 0 \quad\left(z, w \in \mathbb{B}^{d}\right) .
$$

The Nevanlinna-Pick problem for these functions (in the operator-valued version) has been recently considered in [6. It was shown that every solution of the problem corresponds to a unitary extension of a partially defined isometric operator, which led to a parametrization of all solutions given in terms of a Redheffer linear fractional transformation. We shall pose a more general interpolation problem and, upon including it in the general scheme of Problem 1.1, shall get a different parametrization of all its solutions.

We shall use standard notations: points in $\mathbb{C}^{d}$ will be denoted by $z=\left(z_{1}, \ldots, z_{d}\right)$, where $z_{j} \in \mathbb{C}$ and $\langle z, w\rangle=\sum_{j=1}^{d} z_{j} \bar{w}_{j}$ will stand for the standard inner product in $\mathbb{C}^{d}$. For multiindices $\mathbf{n}=\left(n_{1}, \ldots, n_{d}\right) \in \mathbb{N}^{d}$ we set

$$
n_{1}+n_{2}+\ldots+n_{d}=|\mathbf{n}|, \quad n_{1} ! n_{2} ! \ldots n_{d} !=\mathbf{n} !, \quad z_{1}^{n_{1}} z_{2}^{n_{2}} \ldots z_{d}^{n_{d}}=z^{\mathbf{n}} .
$$

The kernel

$$
\mathbf{k}(z, w)=\frac{1}{1-\langle z, w\rangle}
$$

is positive on $\mathbb{B}^{d}$. It can be shown (see, e.g., [5, Lemma 3.8]) that in the metric of $\mathcal{H}(\mathbf{k})$,

$$
\left\langle z^{\mathbf{n}}, z^{\mathbf{m}}\right\rangle_{\mathcal{H}(\mathbf{k})}= \begin{cases}\frac{\mathbf{n} !}{|\mathbf{n}| !} & \text { if } \mathbf{n}=\mathbf{m}, \\ 0 & \text { otherwise }\end{cases}
$$

which leads to the following characterization of $\mathcal{H}(\mathbf{k})$ :

$$
\mathcal{H}(\mathbf{k})=\left\{f(z)=\sum_{\mathbf{n} \in \mathbb{N}^{d}} f_{\mathbf{n}} z^{\mathbf{n}}, \text { with }\|f\|_{\mathcal{H}(\mathbf{k})}^{2}=\sum_{\mathbf{n} \in \mathbb{N}^{d}} \frac{\mathbf{n} !}{|\mathbf{n}| !}\left|f_{\mathbf{n}}\right|^{2}<\infty\right\} .
$$


The space $\mathcal{H}\left(\mathbf{k} I_{p}\right)$ can be viewed as the tensor product Hilbert space $\mathcal{H}(\mathbf{k}) \otimes \mathbb{C}^{p \times 1}$ and we denote it $\mathcal{H}^{p}(\mathbf{k})$ for short. Similarly, we use the notation $\mathcal{H}^{p \times q}(\mathbf{k})$ for the space of $\mathbb{C}^{p \times q}$-valued functions with entries in $\mathcal{H}(\mathbf{k})$. Note that the bilinear form defined in (2.4) takes in this context the form

$$
[H, F]_{\mathcal{H}(\mathbf{k})}=\sum_{\mathbf{n} \in \mathbb{N}^{d}} \frac{\mathbf{n} !}{|\mathbf{n}| !} F_{\mathbf{n}}^{*} H_{\mathbf{n}}
$$

and makes sense for every choice of $H \in \mathcal{H}^{p \times m}(\mathbf{k})$ and $F \in \mathcal{H}^{p \times \ell}(\mathbf{k})$.

The kernel $K_{S}$ defined in (3.1) is a particular case of (1.5) corresponding to the particular choice of $K^{(1)}=\mathbf{k} I_{q}$ and $K^{(2)}=\mathbf{k} I_{p}$, and condition (3.1) means that $S$ is a contractive multiplier from $\mathcal{H}^{q}(\mathbf{k})$ to $\mathcal{H}^{p}(\mathbf{k})$.

Let matrices $C_{1} \in \mathbb{C}^{p \times m}, C_{2} \in \mathbb{C}^{q \times m}$ and $A_{1}, \ldots, A_{d} \in \mathbb{C}^{m \times m}$ be such that

1. The joint spectrum of $A_{1}, \ldots, A_{d}$ sits inside $\mathbb{B}^{d}$ :

$$
\sigma_{\text {joint }}\left(A_{1}, \ldots, A_{d}\right) \subset \mathbb{B}^{d} .
$$

2. For every two products ("words") $W_{\mathbf{n}}\left(A_{1}, \ldots, A_{d}\right)$ and $W_{\mathbf{n}}^{\prime}\left(A_{1}, \ldots, A_{d}\right)$ containing the same number $n_{j}$ of a letter $A_{j}$ (for all $\left.j=1, \ldots, d\right)$,

$$
\left[\begin{array}{l}
C_{1} \\
C_{2}
\end{array}\right] W_{\mathbf{n}}\left(A_{1}, \ldots, A_{d}\right)=\left[\begin{array}{l}
C_{1} \\
C_{2}
\end{array}\right] W_{\mathbf{n}}^{\prime}\left(A_{1}, \ldots, A_{d}\right)
$$

(for instance, the last condition is clearly satisfied if $A_{\ell} A_{j}=A_{j} A_{\ell}(\ell, j=1, \ldots, d)$ ). We set

$$
C=\left[\begin{array}{c}
C_{1} \\
C_{2}
\end{array}\right], \quad \mathbf{A}=\left[\begin{array}{c}
A_{1} \\
\vdots \\
A_{d}
\end{array}\right] \quad \text { and } Z(z)=\left[\begin{array}{llll}
z_{1} I_{m} & z_{2} I_{m} & \ldots & z_{d} I_{m}
\end{array}\right],
$$

and we shall use the shorthand notation

$$
C \mathbf{A}^{\mathbf{n}}=C A_{1}^{n_{1}} A_{2}^{n_{2}} \cdots A_{d}^{n_{d}}
$$

for a multiindex $\mathbf{n}=\left(n_{1}, n_{2}, \ldots, n_{d}\right)$, when the order of multipliers is not essential. We assume furthermore that

3. The series $\sum_{\mathbf{n} \in \mathbb{N}^{d}} \frac{|\mathbf{n}| !}{\mathbf{n} !}\left(\mathbf{A}^{\mathbf{n}}\right)^{*} C^{*} C \mathbf{A}^{\mathbf{n}}$ converges.

Note that the assumptions 1 and 3 provide that the function

$$
G(z)=\left(I_{m}-Z(z) \mathbf{A}\right)^{-1}
$$

is analytic in $\overline{\mathbb{B}}_{d}$ and belongs to $\mathcal{H}^{m \times m}(\mathbf{k})$. Making use of notation (3.8) one can write

$$
C G(z)=C\left(I_{m}-\sum_{j=1}^{d} z_{j} A_{j}\right)^{-1}=C \sum_{\mathbf{n} \in \mathbb{N}^{d}} \frac{|\mathbf{n}| !}{\mathbf{n} !} \mathbf{A}^{\mathbf{n}} z^{\mathbf{n}}
$$

The symbol $\mathbf{I P}(\mathbf{A}, C)$ will be used to denote the following interpolation problem:

Given $\mathbf{A}, C$ and $G$ as above, find necessary and sufficient conditions which insure the existence of a function $S \in \mathcal{S}^{p \times q}$ such that

$$
\mathbf{M}_{S}^{*}\left(C_{1} G(z)\right)=C_{2} G(z)
$$

and describe the set of all such functions. 
Remark 3.1. It can be easily seen that the particular choice of

$$
\left[\begin{array}{l}
C_{1} \\
C_{2}
\end{array}\right]=\left[\begin{array}{lll}
b_{1} & \ldots & b_{m} \\
c_{1} & \ldots & c_{m}
\end{array}\right], \quad A_{j}=\left[\begin{array}{ccc}
\bar{w}_{1}^{(j)} & & \\
& \ddots & \\
& & \bar{w}_{m}^{(j)}
\end{array}\right] \quad(j=1, \ldots, d)
$$

in (3.11) for prescribed $m$ points $w^{(\ell)}=\left(w_{1}^{(\ell)}, \ldots, w_{d}^{(\ell)}\right) \in \mathbb{B}^{d}$ and vectors $b_{\ell} \in \mathbb{C}^{p}$, $c_{\ell} \in \mathbb{C}^{q}$, leads to left-sided Nevanlinna-Pick conditions $b_{\ell}^{*} S\left(w^{(\ell)}\right)=c_{\ell}^{*} \quad(\ell=$ $1, \ldots, m)$.

The $\operatorname{IP}(\mathbf{A}, C)$ can be included in the general scheme of Problem 1.1 upon setting

$$
H(z)=C_{1} G(z) \in \mathcal{H}^{p \times m}(\mathbf{k}) \quad \text { and } \quad F(z)=C_{2} G(z) \in \mathcal{H}^{q \times m}(\mathbf{k}) .
$$

Then the function $B$ defined in (2.6) takes the form $B(z)=\left(C_{1}-S(z) C_{2}\right) G(z)$. By Theorem 2.4 we get that a $\mathbb{C}^{p \times q}$-valued function $S$ analytic in $\mathbb{B}^{d}$ is a soluttion to the $\mathbf{I P}(\mathbf{A}, C)$ if and only if

$$
\mathbf{K}(z, w):=\left[\begin{array}{cc}
P & G(\omega)^{*}\left(C_{1}^{*}-C_{2}^{*} S(w)^{*}\right) \\
\left(C_{1}-S(z) C_{2}\right) G(z) & \frac{I_{p}-S(z) S(w)^{*}}{1-\langle z, w\rangle}
\end{array}\right] \succeq 0 \quad\left(z, w \in \mathbb{B}^{d}\right),
$$

where

$$
P:=\left[C_{2} G, C_{2} G\right]_{\mathcal{H}(\mathbf{k})}-\left[C_{1} G, C_{1} G\right]_{\mathcal{H}(\mathbf{k})} .
$$

Making use of the signature matrix

$$
J=\left[\begin{array}{cc}
I_{p} & 0 \\
0 & -I_{q}
\end{array}\right]
$$

we can represent $P$ as

$$
P=[J C G, C G]_{\mathcal{H}(\mathbf{k})} .
$$

In contrast to the general case, inequality (3.13) admits a nice description of all its solutions. First we note that the Pick matrix $P$ defined in (3.14) satisfies the generalized Stein equation

$$
P-\sum_{j=1}^{d} A_{j}^{*} P A_{j}=C^{*} J C
$$

Indeed, due to conditions (3.6) one can solve (3.17) iteratively to get a (unique) solution $P$ of (3.17) in the form of a uniformly converging series

$$
P=\sum_{\mathbf{n} \in \mathbb{N}^{d}} \frac{|\mathbf{n}| !}{\mathbf{n} !}\left(\mathbf{A}^{\mathbf{n}}\right)^{*} C^{*} J C \mathbf{A}^{\mathbf{n}} .
$$

On the other hand, substituting the Taylor expansion (3.10) for $G$ into the left-hand side in (3.16) and making use of (3.4) we come to the same expression for $P$ :

$$
\begin{aligned}
{[J C G, C G]_{\mathcal{H}(\mathbf{k})} } & =\left[J C \sum_{\mathbf{n} \in \mathbb{N}^{d}} \frac{|\mathbf{n}| !}{\mathbf{n} !} \mathbf{A}^{\mathbf{n}} z^{\mathbf{n}}, C \sum_{\mathbf{n} \in \mathbb{N}^{d}} \frac{|\mathbf{n}| !}{\mathbf{n} !} \mathbf{A}^{\mathbf{n}} z^{\mathbf{n}}\right]_{\mathcal{H}(\mathbf{k})} \\
& =\sum_{\mathbf{n} \in \mathbb{N}^{d}} \frac{|\mathbf{n}| !}{\mathbf{n} !}\left(\mathbf{A}^{\mathbf{n}}\right)^{*} C^{*} J C \mathbf{A}^{\mathbf{n}} .
\end{aligned}
$$


Let us assume that $P$ is positive definite. Then the $\left.\mathbb{C}^{(p+q) \times(m d+p+q)}\right)$-valued function

$$
\begin{aligned}
& \Theta(z)=\left[\begin{array}{ll}
0 & I_{p+q}
\end{array}\right] \\
& +C G(z) P^{-1}\left[\begin{array}{llll}
\left(z_{1} I_{m}-A_{1}^{*}\right) P^{\frac{1}{2}} & \ldots & \left(z_{d} I_{m}-A_{d}^{*}\right) P^{\frac{1}{2}} & -C^{*} J
\end{array}\right]
\end{aligned}
$$

is analytic in $\mathbb{B}^{d}$ and satisfies

$$
\frac{J-\Theta(z) \mathbf{J} \Theta(w)^{*}}{1-\langle z, w\rangle}=C G(z) P^{-1} G(w)^{*} C^{*}, \quad \text { where } \quad \mathbf{J}=\left[\begin{array}{cc}
I_{m d} & 0 \\
0 & J
\end{array}\right],
$$

for every choice of $z=\left(z_{1}, \ldots, z_{d}\right)$ and $w=\left(w_{1}, \ldots, w_{d}\right)$ in $\mathbb{B}_{d}$. Indeed, it follows readily from (3.19) that

$$
J-\Theta(z) \mathbf{J} \Theta(w)^{*}=C G(z) P^{-1} T(z, w) P^{-1} G(w)^{*} C,
$$

where

$$
\begin{aligned}
T(z, w)= & \left(I-\sum_{j=1}^{d} \bar{w}_{j} A_{j}^{*}\right) P+P\left(I-\sum_{j=1}^{d} z_{j} A_{j}\right) \\
& -\sum_{j=1}^{d}\left(z_{j} I-A_{j}^{*}\right) P\left(\bar{w}_{j} I-A_{j}\right)-C^{*} J C .
\end{aligned}
$$

Making use of (3.17), we get

$$
T(z, w)=2 P-\sum_{j=1}^{d}\left(z_{j} \bar{w}_{j} P+A_{j}^{*} P A_{j}\right)-C^{*} J C=(1-\langle z, w\rangle) P,
$$

which together with (3.20) imply (3.19).

Still assuming that $P$ is positive definite, we conclude that $(3.13)$ is equivalent to

$\frac{I_{p}-S(z) S(w)^{*}}{1-\langle z, w\rangle}-\left(C_{1}-S(z) C_{2}\right) G(z) P^{-1} G(\omega)^{*}\left(C_{1}^{*}-C_{2}^{*} S(w)^{*}\right) \succeq 0 \quad\left(z, w \in \mathbb{B}^{d}\right)$,

which in its turn, can be written as

$$
\left[\begin{array}{ll}
I_{p} & -S(z)
\end{array}\right]\left\{\frac{J}{1-\langle z, w\rangle}-C G(z) P^{-1} G(w)^{*} C\right\}\left[\begin{array}{c}
I_{p} \\
-S(w)^{*}
\end{array}\right] \succeq 0 \quad\left(z, w \in \mathbb{B}^{d}\right) .
$$

Taking advantage of (3.19), we rewrite the last inequality as

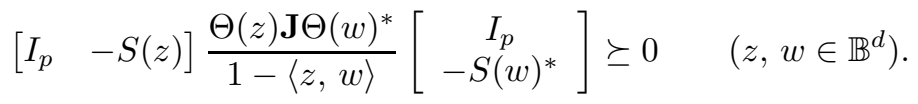

Theorem 3.2. Let $P$ be positive definite and let

$$
\Theta=\left[\begin{array}{ll}
\Theta_{11} & \Theta_{12} \\
\Theta_{21} & \Theta_{22}
\end{array}\right]:\left[\begin{array}{l}
\mathbb{C}^{m d+p} \\
\mathbb{C}^{q}
\end{array}\right] \rightarrow\left[\begin{array}{l}
\mathbb{C}^{p} \\
\mathbb{C}^{q}
\end{array}\right]
$$

be the partition of the function $\Theta$ given by (3.18) into four blocks of the indicated sizes. Then the set of all solutions $S$ of the $\mathbf{I P}(\mathbf{A}, C)$ are parametrized by the linear fractional transformation

$$
S(z)=\left(\Theta_{11}(z) \mathcal{E}(z)+\Theta_{12}(z)\right)\left(\Theta_{21}(z) \mathcal{E}(z)+\Theta_{22}(z)\right)^{-1},
$$

when the parameter $\mathcal{E}$ varies on the set $\mathcal{S}^{(m d+p) \times q}$. 
Proof. It follows from (3.19) and (3.22) that

$$
-I_{q}-\Theta_{21}(z) \Theta_{21}(z)^{*}+\Theta_{22}(z) \Theta_{22}(z)^{*} \geq 0 \quad\left(z \in \mathbb{B}^{d}\right) .
$$

Therefore, $\Theta_{22}(z)$ is invertible at every point $z \in \mathbb{B}^{d}$ and $\left\|\Theta_{22}(z)^{-1} \Theta_{21}(z)\right\|<1$. Then the function

$$
\Theta_{21}(z) \mathcal{E}(z)+\Theta_{22}(z)=\Theta_{22}(z)\left(\Theta_{22}(z)^{-1} \Theta_{21}(z) \mathcal{E}(z)+I_{q}\right)
$$

is invertible in $\mathbb{B}^{d}$ for every $\mathcal{E} \in \mathcal{S}^{(m d+p) \times q}$, which means that the transformation (3.2) is well defined on the set $\mathcal{S}^{(m d+p) \times q}$.

According to the preceding analysis $S$ is a solution of the $\operatorname{IP}(\mathbf{A}, C)$ if and only if it satisfies the inequality (3.21). Setting

$$
[u(z)-v(z)]=\left[\begin{array}{ll}
I_{p} & -S(z)
\end{array}\right] \Theta(z)
$$

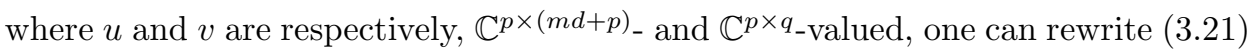
as

$$
\frac{u(z) u(w)^{*}-v(z) v(w)^{*}}{1-\langle z, w\rangle} \succeq 0 \quad\left(z, w \in \mathbb{B}^{d}\right),
$$

which is equivalent (see, e.g., [3] Theorem 3.1]) to the existence of a function $\mathcal{E} \in$ $\mathcal{S}^{(m d+p) \times q}$ such that

$$
u(z) \mathcal{E}(z)=v(z) \quad\left(z \in \mathbb{B}^{d}\right) .
$$

By (3.24), we conclude that $S$ is a solution of the $\operatorname{IP}(\mathbf{A}, C)$ if and only if

$$
\left[\begin{array}{ll}
I_{p} & -S(z)
\end{array}\right] \Theta(z)=u(z)\left[\begin{array}{ll}
I_{m d+p} & -\mathcal{E}(z)
\end{array}\right]
$$

for some bounded analytic function $u$ and a function $\mathcal{E} \in \mathcal{S}^{(m d+p) \times q}$. The latter is equivalent to

$$
\left[\begin{array}{ll}
I_{p} & -S(z)
\end{array}\right] \Theta(z)\left[\begin{array}{c}
\mathcal{E}(z) \\
I_{q}
\end{array}\right]=0
$$

which, being rewritten as

$$
\Theta_{11}(z) \mathcal{E}(z)+\Theta_{12}(z)-S(z)\left(\Theta_{21}(z) \mathcal{E}(z)+\Theta_{22}(z)\right)=0,
$$

is evidently equivalent to (3.23).

As a consequence of the last theorem we get that under the assumption $P>0$, the $\mathbf{I P}(\mathbf{A}, C)$ has infinitely many solutions. Using the standard approximation argument it can be easily shown that if $P$ is positive semidefinite, then there exists a solution to the $\mathbf{I P}(\mathbf{A}, C)$ (the questions about paremetrization of all solutions and uniqueness criteria are more delicate and will be considered elsewhere). Therefore, the condition $P \geq 0$ is necessary and sufficient for the $\operatorname{IP}(\mathbf{A}, C)$ to be solvable (the necessity of this condition follows readily from (3.13)). This conclusion is not surprising in light of recent papers [1], [14, [12], [13], [6] where this result has been established (for a wide class of reproducing kernel Hilbert spaces) for the Nevanlinna-Pick problem.

I am indebted to the referee whose suggestions led to a substantial simplification of the proof of Theorem 2.4. 


\section{REFERENCES}

1. J. Agler and J. E. McCarthy, Complete Nevanlinna-Pick kernels, J. Funct. Anal., 175 (2000), 111-124. MR 2001h:47019

2. D. Alpay and V. Bolotnikov, On tangential interpolation in reproducing kernel Hilbert modules and applications, in: Topics in Interpolation Theory (H. Dym, B. Fritzsche, V. Katsnelson and B. Kirstein, eds.), Oper. Theory Adv. Appl., OT95, Birkhäuser Verlag, Basel, 1997, pp. 37-68. MR 99b:30055

3. D. Alpay, V. Bolotnikov and H. T. Kaptanoğlu, The Schur algorithm and reproducing kernel Hilbert spaces in the ball, Linear Algebra Appl., 342 (2002), 163-186. MR 2002m:47019

4. N. Aronszajn, Theory of reproducing kernels, Trans. Amer. Math. Soc., 68 (1950), 337-404. MR 14:479c

5. W. Arveson, Subalgebras of $C^{*}$-algebras. III. Multivariable operator theory, Acta Math. 181 (1998), no. 2, 159-228. MR 2000e:47013

6. J. A. Ball, T. T. Trent and V. Vinnikov, Interpolation and commutant lifting for multipliers on reproducing kernels Hilbert spaces, Oper. Theory Adv. Appl. 122 (2001), 89-138. MR 2002f: 47028

7. V. Bolotnikov and H. Dym, On boundary interpolation for matrix Schur functions, Preprint, 1999.

8. L. de Branges and J. Rovnyak, Square summable power series, Holt, Rinehart and Winston, New York, 1966. MR 35:5909

9. F. Beatrous and J. Burbea, Positive-definiteness and its applications to interpolation problems for holomorphic functions, Trans. Amer. Math. Soc., 284 (1984), no.1, 247-270. MR 85e:32020

10. H. Dym, $J$ contractive matrix functions, reproducing kernel spaces and interpolation, CBMS Lecture Notes, vol. 71, Amer. Math. Soc., Rhode Island, 1989. MR 90g:47003

11. I. V. Kovalishina and V. P. Potapov, Seven Papers Translated from the Russian, Amer. Math. Soc. Transl. (2), 138, Providence, R.I., 1988. MR 89f:00030

12. S. McCullough, The local de Branges-Rovnyak construction and complete Nevanlinna-Pick kernels, in Algebraic methods in operator theory (Ed. R. Curto and P. E. T. Jorgensen), Birkhäuser-Verlag, Boston, 1994, pp. 15-24. MR 95j:47016

13. G. Popescu, Interpolation problems in several variables, J. Math. Anal. Appl., 227 (1998), 227-250. MR 99i:47028

14. P. Quiggin, For which reproducing kernel Hilbert spaces is Pick's theorem true?, Integral Equations Operator Theory 16 (1993), no. 2, 244-266. MR 94a:47026

15. D. Sarason, Sub-Hardy Hilbert spaces in the unit disk, John Wiley and Sons Inc., New York, 1994. MR 96k:46039

Department of Mathematics, The College of William and Mary, Williamsburg, VirGINIA 23187-8795

E-mail address: vladi@math.wm.edu 